\title{
Developing a Model and Validating an Instrument for Measuring the Adoption and Utilisation of Mobile Government Services Adoption in Saudi Arabia
}

\author{
Mohammed Alonazi \\ University of Sussex \\ Informatics Department \\ Brighton, UK \\ Email: M.Alonazi@sussex.ac.uk
}

\author{
Natalia Beloff \\ University of Sussex \\ Informatics Department \\ Brighton, UK \\ Email: N.Beloff@sussex.ac.uk
}

\author{
Martin White \\ University of Sussex \\ Informatics Department \\ Brighton, UK \\ Email: M.White@sussex.ac.uk
}

\begin{abstract}
Many governments worldwide are taking advantage of the latest developments in mobile technology to take the digital delivery of government information and services (egovernment) to their citizens a stage further. Accessing government information and services via a mobile device is known as m-government, a system designed to serve citizens, companies and government agencies alike. M-government also has unique advantages over e-government, not least enabling users to access government services at any time and from any location. This paper presents a pilot study of the MGAUM model that was developed to analyze factors influencing the adoption rate of m-government services in Saudi Arabia. With the aim of validating a survey instrument with which to conduct the main study in Saudi Arabia, a pilot survey instrument was developed and modified by using previous instruments from research into both e-government and mgovernment. This pilot questionnaire was distributed to 71 Saudi citizens in the UK, and the final sample was 58 valid responses. The results of this pilot study reveal that all items in the survey instrument were reliable and valid within the range of acceptable academic research and suitable for use in the main study. The results of this pilot study were analyzed using SPSS.
\end{abstract}

\section{INTRODUCTION}

$\mathrm{T}$ he revolution in Information and Communication Technologies (ICT) has resulted in governments across world digitizing their services to citizens through tools such as mobile technologies and the Internet. Arguably, the implementation of e-government has resulted in better communication between citizens and their governments with enhanced access to services and information as well as improvements in government transparency, accountability and public governance[1], [2], [3]. Several governments have also implemented mobile government (m-government) by developing innovative service delivery channels that utilize wireless and mobile technologies [4], [5]. Accordingly, in this study, m-Government is defined as the use of mobile technology to deliver and improve e-government services and information to citizens, businesses and all government agencies. Previous studies have identified m-government as both separate to e-government or as an as an extension or replacement of it [5], [6]. Kushchu \& Kuscu stress that it is an advance in government service delivery as it offers the public a valuable extra means to access services and information[4]. Although the same principles are common to IEEE Catalog Number: CFP1985N-ART (C)2019, PTI e-government and m-government, the latter is distinguished by features that are particular to it:

- The main advantage for citizens of m-government is its mobility, giving citizens access to the network at any time and from anywhere[7].

- As mobile phones can be used easily, citizens can instantly receive messages from government service providers[8].

- In many countries, the mobile phone has recently become the primary way people communicate over distance; the mobile phone has thus arguably become a part of everyday life for many people[9], [10]. For this reason, being able to access government services via mobile devices might be the best route for citizens.

- More and more people are using their mobiles to access the web. For citizens, this means they neither have to visit the service provider in person nor go home to use their computer when they want to access government services and information[8], [10].

- Access to the Internet may depend on a country's economy in terms of the extent of Internet access and how many citizens have access to computers[6],[4]; these limitations can be overcome by providing mobile services.

Therefore, by using an m-government system provided by wireless technology, citizens will acquire opportunities for personalized, real-time access to government services and information[11], [7]. Users in remote areas will especially benefit from being able to access government services and information as m-services have the advantage of being affordable, easy and immediate to access and require a relatively low level of digital literacy to operate successfully [12], [7]. Given these characteristics, the adoption of an m-government system has benefits for citizens and government alike. Any government services that citizen can use via mobile devices, such as apps, mobile website (web platform), SMS or call centre, are considered as mobile government services. So, this research focuses on all government services are provided via mobile devices. Therefore, the government agencies should concentrate on 
offering different channels for citizens to conduct government transactions at the same time to allow fast and easy access to these services fast. However, the main difference between the website and mobile devices is that features on the mobile web platform and Apps that add value to services, such as reminders, location and camera, are not found on the website. However, from the researcher's experience, people prefer to use native applications rather than a mobile responsive website.

The goals of this research are to investigate and understand Saudi citizens' perceptions towards the adoption and utilization of m-government services in developing countries, particularly Saudi Arabia, in order to increase the adoption rate of $\mathrm{m}$-government services. This paper focused on validating the MGAUM model that has been developed by integrating the TAM model in conjunction with social, cultural and technological factors that the available literature has suggested are key to understanding technology acceptance within a specific context. The results of this pilot study reveal that all items in the survey instrument were reliable and valid within the range of acceptable academic research and suitable for use in the main study.

\section{BACKGROUND AND CONTEXT}

Despite m-government systems being available for several years, citizens' adoption of e-government services in general and m-government services in particular still falls below expectations [1],[2],[13],[14]. Furthermore, in Saudi Arabia, like in most developing countries, m-government implementation is still in its infancy and there are many challenges related to implementation, adoption and use $[1],[15],[16]$. Factors such as the rate of mobile device and Internet penetration and their security, reliability and effectiveness, affect how successful a government will be at implementing m-government and user adoption and accounts for global variation [11]. However, there is a lack of research that allows a clear understanding of how factors such as these might impact the adoption and use of mgovernment services. This study rectifies this problem by providing a theoretical model purposely developed to carry out empirical research in this area. The results of this research will yield new insights about the key factors influencing the adoption of Saudi m-government, which will be invaluable to policy makers who require strategies that will result in faster and more efficient adoption of $\mathrm{m}$ government services; as well as providing new information for researchers in the field and the ICT industry.

Research carried out in a number of different areas such as Malaysia and rural China [12], [17],[18] have made use of adaptations of the Technology Acceptance Model (TAM) and provided examples of how a number of social, cultural and technical factors can usefully be included in the TAM to provide insights into the influences on citizens' intention to utilize m-government systems to access services and information. Cultural and technological factors like culture, trust and lack of necessary infrastructure have been demonstrated to be significant by comparative studies of $\mathrm{m}$ government adoption in developing and developed countries [19],[20].

The adoption of m-government in Arab countries, however, still requires further research. Studies conducted in these areas [21],[22],[23],[20],[24] have revealed that factors such as trust, citizens' perceptions of the compatibility of mgovernment with their lifestyles, culture, awareness and the quality of the system are significant. Further, these studies show that there have been no empirical studies of $\mathrm{m}$ government adoption in Saudi Arabia that includes factors like compatibility or culture. Similarly, there are no studies that take the quality of both technical and human factors into account or that investigate the issues from the viewpoint of the providers in addition to the intended users. There is clearly a need to carry out further research into Saudi mgovernment adoption.

In order to analyze factors that affect users' adoption and use of m-government, the researcher has developed a model called the Mobile Government Adoption and Utilization Model (MGAUM)[25]. The remainder of this paper consists of the following sections: Section 3 outlines the MGAUM, Section 4 covers the research methodology; Section 5 contains a descriptive and statistical analysis of the findings and Section 6 is the conclusion.

\section{THEPROPOSED RESEARCH MODEL (MGAUM)}

In order to analyze factors that affect users' adoption and use of m-government, the researcher has developed a model called the Mobile Government Adoption and Utilization Model (MGAUM)[25]. MGAUM has been developed based on a critical analysis of the literature that relates to acceptance of technology, in conjunction with insights from several models and theories that are commonly used to analyze acceptance and usage of technologies. MGAUM integrates the Technology Acceptance Model [26],with a number of social, cultural and technological factors, taken from other recognized theoretical acceptance models that have been identified as key factors in the literature.

Further, MGAUM contains one dependent variable namely: Intention to use m-government (ITU), and three groups of independent variables namely: Practical Factors (PF), Human Factors (HF), and Technical Factors (TF). These independent variables comprise the key factors that critically influence the adoption and use of m-government.

This model uses the TAM as a starting point along with factors from other theories to analyze how users adopt m- 
government services. Two factors were taken from the TAM: Perceived Ease of Use (PEOU) and Perceived Usefulness (PU). These two factors comprise the Practical Factors of MGAUM; the Human Factors are those that were revealed by the literature as important in further understanding the role played by the individual users' acceptance in this context and the Technical Factors are those identified as most likely to affect the adoption of mgovernment. The final design of MGAUM also used the researcher's experience of the local problems of accessing government services and information in Saudi Arabia to select those factors identified as key in the relevant literature. The main aim of MGAUM is to investigate the adoption of m-government services by citizens in developing countries, particularly Saudi Arabia, in order to increase the adoption rate of m-government services. The research model MGAUM is shown in Fig. 1.

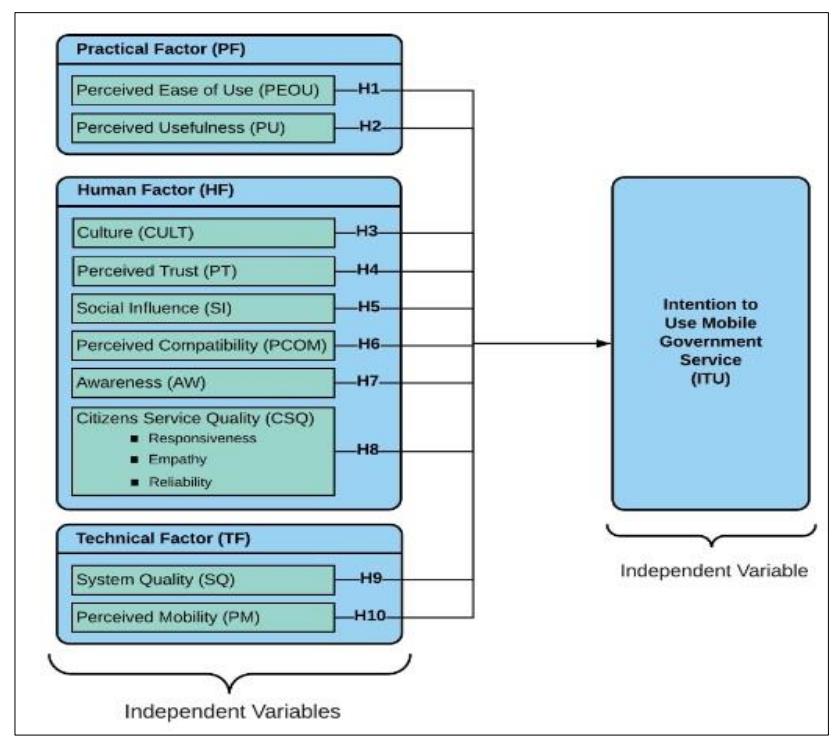

Fig. 1: The research model (MGAUM) [25].

The hypotheses of the study are as follows[25]:

H1: Perceived Ease of Use positively influences intention to use m-government services.

H2: Perceived Usefulness positively influences intention to use m-government services.

H3: Culture influences intention to use m-government services.

H4: Trust positively influences intention to use mgovernment services.

H5: Social Influence affects intention to use m-government services.

H6: Compatibility positively influences intention to use mgovernment services.

H7: Awareness positively influences intention to use mgovernment services.
H8: Citizen service quality factors (responsiveness, empathy and reliability) positively influence intention to use m-government services.

H9: System Quality positively influences intention to use m-government services.

H10: Perceived Mobility positively influences intention to use m-government services.

\section{THE RESEARCH METHODOLOGY}

This pilot study was conducted in the United Kingdom. The questionnaire was distributed to Saudi citizens (public users) whether they had used mobile government services or not. Participants had to be 18 years or older to participate in this survey. Seventy-one participants took part in the questionnaire; but as all the questions had to be answered as they represented the research model, incomplete questionnaires were excluded from the survey. Therefore, the total valid responses constituted a sample of 58 Saudi citizens. The pilot study data were analysed by using the SPSS program.

The survey questionnaire contains 76 items and all the questions were developed and modified from instruments used in previous research into both E-government and Mgovernment. All items were measured with a 5-point Likert scale from 'strongly agree' to 'strongly disagree'. In order to collect enough data to ensure a thorough analysis, the questionnaire had to be relatively lengthy. Manual distribution meant that the researcher could clarify points for the participants if necessary and contributed to obtaining a higher response rate.

\section{A. Reliability and validity of the study}

If a research instrument is to be reliable, it should produce similar results if used in comparable conditions [27]. Furthermore, an instrument's reliability depends on how free it is of error [28]. In order to assess the reliability of the questionnaire used in the pilot study, Cronbach's Alpha was chosen because the internal consistency of the constructs used in the questionnaire had to be established; in other words, we needed to measure the extent to which items in the questionnaire measured the same things when referring to a specific independent or dependent construct, and how these related to each other. Cronbach's Alpha is the most commonly used test to calculate and evaluate internal consistency, and thus reliability [29].Cronbach's Alpha has a scale of 0 to 1 , with 1 being the highest reliability, a value of independent and dependent variables 0.6 is considered to be acceptable [30]. Table 1 indicates the Cronbach's Alpha results for the complete pilot study instrument, which demonstrates that the reliability of each of the constructs (independent and dependent variables) lies within the range of what is considered acceptable in academic research.

A valid research instrument measures what the researcher intended [31], and the validity of an instrument is the extent to which it does this and provides the information required [32]. In order to establish the validity of our research instrument, the face validity and content validity methods 
were selected rather than construct validity and criterion validity. This method is designed to establish the extent to which the purpose of the instrument is clear even to the lay person with only basic education [33], for example a 1st grader at school. There is a high level of face validity if the items in the questionnaire are clear and unambiguous, if the items are perceived as difficult to understand or confusing, then the face validity is low [33]. The pilot study allowed the face validity of each item to be tested by participants so as to ensure that the model's factors measured what they were intended to measure. Items without sufficient clarity, unambiguity or relevance were revised or deleted accordingly. Furthermore, six academics, all of whom had expertise in the field, were asked to review the items in the research instrument, this review and the pilot study demonstrated that all the items used therefore had a good degree of content validity.

TABLE1: INTERNAL CONSISTENCY OF THE PILOT STUDY INSTRUMENT

\begin{tabular}{|l|c|c|}
\hline \multicolumn{1}{|c|}{ Measured Variable } & No. of Items & Cronbach's Alpha \\
\hline Perceived Usefulness (PU) & 7 & .841 \\
\hline Perceived Ease of Use (PEOU) & 4 & .848 \\
\hline Social Influence(SI) & 3 & .753 \\
\hline Perceived Compatibility (PCOM) & 2 & .824 \\
\hline Perceived Trust (PT) & 7 & .618 \\
\hline Culture (CULT) & 5 & .606 \\
\hline Awareness (AW) & 4 & .816 \\
\hline Perceived Mobility (AW) & 3 & .819 \\
\hline Citizens Service Quality (CSQ) & 8 & .920 \\
\hline System Quality (SQ) & 7 & .755 \\
\hline Intention to use (ITU) & 4 & .879 \\
\hline
\end{tabular}

\section{DESCRIPTIVE AND STATISTICAL ANALYSIS}

\section{A. Respondents' demographic data}

In this study, 58 participants answered the questionnaire, and that 38 participants were male $(65.5 \%)$ and 20 were female $(34.5 \%)$. The highest percentage of participants was in the 31-45 age group; the largest number held a Bachelor degree and over half the participants (51.7\%) were government employees. All participants had smartphone devices, with a large majority of participants using mobiles and the Internet in daily life (93.1\% and $94.8 \%$ respectively). Approximately three-quarter of the respondents $(74.1 \%)$ have some knowledge about m-government services in Saudi Arabia, whereas $25.9 \%$ had no knowledge. Moreover, the majority of participants (86.2\%) already used M-government services, but $13.8 \%$ had never used it. Also, the survey asked the participants that already used m-government services to rate their general experience. The result showed that $44.4 \%$ were very satisfied with m-government services, $52 \%$ were satisfied to some extent, with only $4 \%$ not satisfied with mgovernment services. Furthermore, $27.6 \%$ of participants reported that the requirements of the intended m-government services were not clear and $34.5 \%$ of them reported that the system quality of m-government services was not good.

When asked about what advertising methods could affect awareness of m-government services and encourage their use, participants rated them as follows: social media, emails and text messages (20.17\%), advertisements in public areas (17.54\%), TV and Radio channels (17.1\%), government agencies' websites $(14.03 \%)$ and finally, newspapers and magazines $(14.03 \%)$.

\section{B. The Correlation Analysis}

A correlation coefficient analysis was run on this pilot study to discover the relationships between all constructs and to establish their significance. In 1988, Cohen proposed a guideline for correlation coefficient values as follows: Strong $\mathrm{r}=.50$ to 1.0 , Moderate $\mathrm{r}=.30$ to .49 and Weak $\mathrm{r}=.10$ to .29 [34]. The correlation coefficients can be seen in Table 2, which shows there is a positive relation between the intention to use m-government (Dependent variable ITU) construct and the rest of the constructs, with differences in the strength of this relationship from one construct to another. For instance, there is a strong correlation between Perceived Usefulness and Perceived Compatibility with Intention to use mgovernment services, with moderate correlation for Perceived Ease of Use, Social Influence and Culture whereas there was only a low correlation for Perceived Trust, Awareness, Perceived Mobility, Citizens Service Quality and System Quality.

TABLE 2 :THE CORRELATION BETWEEN THE VARIABLES

\begin{tabular}{|c|c|c|c|c|c|c|c|c|c|c|c|}
\hline \multicolumn{12}{|c|}{ Correlations } \\
\hline & INT & PU & PEOU & SI & PCOM & $\mathrm{PT}$ & CULT & $A W$ & PM & $\operatorname{cs} Q$ & $S Q$ \\
\hline Pearson Correlation & 1 & $.682 * *$ & $.479 * *$ & $.411 * *$ & $.598 *$ & .247 & $.323^{*}$ & .089 & .251 & .074 & $.279 *$ \\
\hline INT Sig. (2-tailed) & & .000 & .000 & .001 & .000 & .062 & .013 & .506 & .057 & .581 & .034 \\
\hline $\mathrm{N}$ & 58 & 58 & 58 & 58 & 58 & 58 & 58 & 58 & 58 & 58 & 58 \\
\hline \multicolumn{12}{|c|}{ ** Correlation is significant at the 0.01 level (2-tailed). } \\
\hline \multicolumn{12}{|c|}{${ }^{*}$. Correlation is significant at the 0.05 level (2-tailed). } \\
\hline
\end{tabular}

The correlation results show that user experience is the first step in adoption; and if a user finds m-Government services easy to use and that it saves time, effort and is compatible with lifestyle and mobile devices, then this impacts positively on his/her behavioural intention to adopt and use them. If services are easy to use, and people do not have to rely on asking for help from another person to use mgovernment service, the number of users will increase. Thus, PU, PEOU and PCOM are essential factors in this study.

The result also indicated that government agencies should focus on the following factors (PT, CULT, AW, PM, CSQ, SI and SQ) to motivate and increase citizens' intention to adopt and use m-government services. Therefore, the government needs to raise awareness about the main goals of $\mathrm{m}$-Government, the availability $\mathrm{m}$-Government services and 
the advantages and benefits gained from the use of $\mathrm{m}$ Government services to conduct various transactions. Public awareness could be enhanced in various ways including interactive advertising and social media campaigns as well as traditional advertising methods such as brochures and advertisements on TV, public transport and in newspapers.

The pilot study has demonstrated that the initial design of the questionnaire will be suitable for the main study to be carried out in situ in Saudi Arabia. The result of the main study will be compared to the pilot study result for extra validation.

\section{CONCLUSION}

This pilot study focused on validating the MGAUM model that has been developed by integrating the TAM model in conjunction with social, cultural and technological factors that the available literature has suggested are key to understanding technology acceptance within a specific context. We intended in this study to provide a valuable insight into the main factors that influence adoption of $\mathrm{m}$ government services in Saudi Arabia, which will be useful for researchers, the ICT industry and for policymakers who are keen to find strategies that result in quicker and more efficient take-up of such services. The reliability and validity of the pilot study have been established. The findings of this pilot study provided us with the basic information and will open the doors for future discussions. We plan to conduct this study in Saudi Arabia with a large number of Saudi citizens to explore what factors encourage them to use m-government services and what the barriers to acceptance are.

\section{REFERENCES}

[1] S. Alghamdi, N. Beloff, Towards a comprehensive model for eGovernment adoption and utilisation analysis: The case of Saudi Arabia, 2014 Fed. Conf. Comput. Sci. Inf. Syst. FedCSIS 2014. 2 (2014) 1217-1225. doi:10.15439/2014F146.

[2] O. Al-Hujran, M.M. Al-Debei, A. Chatfield, M. Migdadi, The imperative of influencing citizen attitude toward e-government adoption and use, Comput. Human Behav. 53 (2015) 189-203. doi:10.1016/j.chb.2015.06.025.

[3] S.Y. Hung, C.M. Chang, S.R. Kuo, User acceptance of mobile egovernment services: An empirical study, Gov. Inf. Q. 30 (2013) 3344. doi:10.1016/j.giq.2012.07.008.

[4] I. Kushchu, M.H. Kuscu, From E-government to M-government: Facing the Inevitable, Proc. 3rd Eur. Conf. EGovernment. (2003) 253260. http://citeseerx.ist.psu.edu.

[5] H.J. Scholl, The mobility paradigm in electronic government theory and practice: A strategic framework, in: Euro Mob. Gov. (Euro MGov) Conf., 2005: pp. 1-10.

[6] S. Alotaibi, D. Roussinov, Developing and Validating an Instrument for Measuring Mobile Government Adoption in Saudi Arabia, World Acad. Sci. Eng. Technol. Int. J. Soc. Behav. Educ. Econ. Bus. Ind. Eng. 10 (2016) 710-716.

[7] M. Ntaliani, C. Costopoulou, S. Karetsos, Mobile government: A challenge for agriculture, Gov. Inf. Q. 25 (2008) 699-716. doi:10.1016/j.giq.2007.04.010.

[8] I. Almarashdeh, M.K. Alsmadi, How to make them use it? Citizens acceptance of M-government, Appl. Comput. Informatics. 13 (2017) 1-6. doi:10.1016/j.aci.2017.04.001.

[9] L.C. Serra, L.P. Carvalho, L.P. Ferreira, J.B.S. Vaz, A.P. Freire, Accessibility Evaluation of E-Government Mobile Applications in Brazil, Procedia Comput. Sci. $67 \quad$ (2015) 348-357. doi:10.1016/j.procs.2015.09.279.
[10] R. Lallana, E-government for development m-government: Mobile/wireless applications, in: 2004.

[11] A.M. Alsenaidy, T. Ahmad, A Review of Current State M Government in Saudi, Glob. Eng. Technol. Rev. (2012) 5-8.

[12] Y. Liu, H. Li, V. Kostakos, J. Goncalves, S. Hosio, F. Hu, An empirical investigation of mobile government adoption in rural China: A case study in Zhejiang province, Gov. Inf. Q. 31 (2014) 432-442. doi:10.1016/j.giq.2014.02.008

[13] R. Alotaibi, L. Houghton, K. Sandhu, Exploring the Potential Factors Influencing the Adoption of M-Government Services in Saudi Arabia : A Qualitative Analysis, 11 (2016) 56-72. doi:10.5539/ijbm.v11n8p56.

[14] N.P. Rana, Y.K. Dwivedi, Citizen's adoption of an e-government system: Validating extended social cognitive theory (SCT), Gov. Inf. Q. 32 (2015) 172-181. doi:10.1016/j.giq.2015.02.002.

[15] S. Alotaibi, D. Roussinov, A conceptual model for examining mobile government adoption in Saudi Arabia, Proc. Eur. Conf. e-Government, ECEG. 2015-Janua (2015) 369-375.

[16] K. Assar, M-government in Saudi Arabia, 5 (2015) 76-83.

[17] T.M. Faziharudean, T. Li-Ly, Consumers' behavioral intentions to use mobile data services in Malaysia, African J. Bus. Manag. 5 (2011) 1811-1821. doi:10.5897/AJBM10.794.

[18] M.A. Alqahtani, R.S. AlRoobaea, P.J. Mayhew, Building a Conceptual Framework for Mobile Transaction in Saudi Arabia: A User " s Perspective Building a Conceptual Framework for Mobile Transaction in Saudi Arabia : A User "s Perspective, (2014) 967-973. doi:10.1109/SAI.2014.6918303.

[19] A. Al-Hadidi, Y. Rezgui, Adoption and Diffusion of m-Government: Challenges and Future Directions for Research, in: IFIP Int. Fed. Inf. Process., 2010: pp. 88-94.

[20] M.A. Shareef, Y.K. Dwivedi, S. Laumer, N. Archer, Citizens' Adoption Behavior of Mobile Government (mGov): A Cross-Cultural Study, Inf. Syst. Manag. 33 (2016) 268-283. doi:10.1080/10580530.2016.1188573.

[21] N.A.S. Almuraqab, M-government adoption factors in the UAE: A partial least-squares approach, Int. J. Bus. Inf. 11 (2017) 404-431. http://ijbi.org/ijbi/article/view/191.

[22] N.A.S. Almuraqab, S.M. Jasimuddin, Factors that Influence EndUsers Adoption of Smart Government Services in the UAE : A Conceptual Framework, 20 (2017) 11-23.

[23] M.A. Shareef, V. Kumar, U. Kumar, Y.K. Dwivedi, e-Government Adoption Model (GAM): Differing service maturity levels, Gov. Inf. Q. 28 (2011) 17-35. doi:DOI: 10.1016/j.giq.2010.05.006.

[24] F.D. Davis, R.P. Bagozzi, P.R. Warshaw, User Acceptance of Computer Technology: a Comparison of Two Theoretical Models., Manag. Sci. Aug1989. 35 (1989) 982-1003. doi:10.2307/2632151.

[25] M. Alonazi, N. Beloff, M. White, MGAUM - Towards a Mobile Government Adoption and Utilization Model: The Case of Saudi Arabia, Int. J. Business, Hum. Soc. Sci. 12 (2018) 459-466.

[26] F.D. Davis, R.P. Bagozzi, P.R. Warshaw, User Acceptance of Computer Technology: a Comparison of Two Theoretical Models., Manag. Sci. Aug1989. 35 (1989) 982-1003. doi:10.2307/2632151.

[27] N. Blunch, Introduction to Structural Equation Modelling Using IBM SPSS Statistics and AMOS", Second Edition, SAGE publication Ltd, 2013.

[28] U. Sekaran, Research methods for business, fourth, John Wiley \& Sons, Inc, 2003. doi:10.1017/CBO9781107415324.004.

[29] E. Eucharia, O. Nnadi, Health Research Design and Methodology, Library of Congress, CRC Press, 1999.

[30] D. Suhr, M. Shay, Guidelines for Reliability , Confirmatory and Exploratory Factor Analysis, in: University of Northern Colorado, Cherry Creek Schools, USA, 2008., 2008: pp. 1-15.

[31] D.K. BHATTACHARYYA, Cross-cultural Management: Texts and Cases., PHI learning private limited, New Delhi, 2010.

[32] D. Colton, R.W. Covert, Designing and Constructing Instruments for Social Research and Evaluation, Jossey-Bass, San Francisco (an imprint of Wiley), 2007.

[33] B. Nevo, FACE VALIDITY REVISITED, J. Educ. Meas. 22 (1985) 287-293. doi:10.1111/j.1745-3984.1985.tb01065.x.

[34] Cohen J., Statistical Power Analysis for the Behavioural Science, 2nd Editio, Routledge, 1988. 